\title{
On irregularities of distribution, IV
}

by

K. F. Rort (London)

Deäicated to the memory of Paul Turan

l. Introduction. Let $z$ be a natural number and let $U_{\theta}^{k+1}, U_{1}^{k+1}$ denote the unit cubes consisting respectively of points $\beta=\left(\beta^{(1)}, \ldots, \beta^{(k+1)}\right)$ with $0 \leqslant \beta^{(j)}<1(j=1, \ldots, k+1)$ and points $a=\left(a^{(1)}, \ldots, a^{(j+1)}\right)$ with $0<\alpha^{(j)} \leqslant 1(j=1, \ldots, k+1)$. Let $\mathscr{P}$ be a finite set in ${U_{0}^{k+1}}^{k+\text { For } a}$ in $U_{1}^{k+1}$, write $Z(\mathscr{P}, B(a))$ for the number of points of $\mathscr{P}$ lying in the box $B(\alpha)$ consisting of all $\beta$ satisfynig $0 \leqslant \beta^{(j)}<\alpha^{(j)}(j=1, \ldots, k+1)$; and pat

$$
D(\mathscr{P} ; B(\alpha))=Z(\mathscr{P} ; B(\alpha))-|\mathscr{P}| \nabla(B(\alpha)),
$$

where $|\mathscr{P}|$ is the number of elements of $\mathscr{P}$ and $T(B(a))$ is the volume of $B(\boldsymbol{a})$.

Roth [5] proved ( $\left.{ }^{1}\right)$ the following result.

THEOREar 1. There exists a positive number $e^{\prime}(\hat{k})$, depending only on $k_{2}$ such that for every $\mathscr{P}$ in $D_{0}^{x+1}$,

$$
\int_{U_{1}^{k+1}} \mid D\left(\mathscr{P} ; B(\alpha)||^{2} d \alpha>\sigma^{\prime}(\hbar)(\log |\mathscr{F}|)^{k} .\right.
$$

The purpose of the present paner is to establish the following complementary result.

THeonem 2. Fror a suitable number $e^{\prime \prime}(k)$ (depending only on $k$ ) there exists, corresponding to every natural number $X \geqslant 2$, a set $\mathscr{P}$ in $U_{0}^{k+1}$ such that $|\mathscr{P}|=N^{T}$ and

$$
\left.\int_{U_{1}^{k+1}}|D| \mathscr{P} ; B(\boldsymbol{a})\right)\left.\right|^{2} d a<c^{\prime \prime}(k)(\log |\mathscr{P}|)^{k}
$$

(1) We use $\int_{U_{1}^{k+1}} d a$ to signify $\int_{U_{1}} \ldots \int_{V_{1}} d a^{(1)} \ldots d \alpha^{(k+1)}$. 
This shows that (apart from the value of the constant) Theorem 1 is best possible.

The case $k+1=2$ of Theorem 2 (the 2 dimensional case) was estab lished by Davenport [1], and different proofs were given by Vilenkin [8], Halton-Zaremba [3] and Roth [6] (but see [7] Appendix for a simplification of this last proof). The case $k+1=3$ was established by Roth in [7], but the method used there fails to generalize to larger $k+1$. Our present result is therefore new when $k+1 \geqslant 4$.

We shall make use of the Hammersley sequence (see [4]) and ideas from Halton's proof of a result (see [2]) which implies that for certain sets $\mathscr{P}$ derived from the Hammersley sequence,

$$
\sup _{\alpha \in O_{1}^{k+1}}|D(\mathscr{P} ; B(\alpha))|<c(k)(\log |\mathscr{P}|)^{k} .
$$

I am indebted to a copy of notes of lectures given by Prof. Wolfgang M. Schmidt (Boulder 1973) for an exposition of the proof of inequalities of type (1.3).

For further discussion and references, see [6], [7].

2. Notation. Although our final result concerns $k+1$ dimensional space, the $k$ dimensional subspace corresponding to the first $k$ coordinates will play an important role. Accordingly, we shall be dealing with both dimensional vectors and $k+1$ dimensional vectors; we reserve bold type for vectors of either kind.

By an "interval" $I$ we shall mean a half-open interval of type $\left[a_{1}, a_{2}\right)$. Thus, for some pair $\alpha_{1}, \alpha_{2}$ satisfying $\alpha_{1}<\alpha_{2}$, the interval $I$ consists of all $\beta$ satisfying $\alpha_{1} \leqslant \beta<\alpha_{2}$.

Suppose $k_{1}=k$ or $k_{1}=k+1$. We shall be concerned with boxes in $k_{1}$ dimensional space of the type consisting of all points $\left(\beta^{(1)}, \beta^{(2)}, \ldots\right.$ $\left.\ldots, \beta^{\left(k_{1}\right)}\right)$ satisfying $\beta^{(j)} \in I^{(j)}\left(j=1,2, \ldots, k_{1}\right)$, where $I^{(j)}$ is an interval $\left[\alpha_{1}^{(j)}, \alpha_{2}^{(j)}\right)$. We shall use the Cartesian product $I^{(1)} \times I^{(2)} \times \ldots \times I^{\left(k_{1}\right)}$ to represent such a box.

We use $R$ to denote a residue class; or, more precisely, the set of all integers in a residue class. Thus, for some natural number $q$ and some integer $a$, the set $R$ consists of all integers congruent to $a$ modulo $q$. For every real $t$, we ase $t+R$ to denote the set $\{t+n ; n \in R\}$.

If $t+R$ has the above meaning and $I$ is an "interval" of the kind described, we define $F[t+R ; X]$ by

$$
F[t+R ; I]=Z(t+R ; I)-q^{-1} l(I)
$$

where $Z(t+R ; I)$ denotes the number of elements of $t+R$ falling into $I$, $q$ is the modulus of the residue class $R$, and $l(I)$ is the length of $I$. We note that

$$
|F[t+R ; I]| \leqslant 1 \quad \text { always. }
$$

3. Preparatory definitions and remarks. Let $h$ be a natural number, and let $p_{1}, p_{2}, \ldots, p_{k}$ be the first $k$ primes. We write

$$
M=M(h)=\left(p_{1} p_{2} \ldots p_{k}\right)^{\hbar} .
$$

For $n=0,1, \ldots, I I-1$ and for ead $j=1,2, \ldots, 7$, we express $n$ in the seale $p_{j}$ by writing

$$
n=\sum_{\nu=0}^{\infty} a_{y}^{(j)} p_{j}^{\nu} \quad\left(0 \leqslant a_{p}<p_{j}\right)_{3}
$$

Where $\Sigma^{\prime}$ signifies that there are only a finite rumber of non-zero terms in the sum. The $a_{p}^{(j)}$ are of course uniquely determined by $n$. We write

$$
x_{n}^{(j)}=p_{j}^{-1} \sum_{j=0}^{\infty} a_{s}^{(j)} p_{j}^{-v}
$$

We note that the $x_{n}^{(j)}$ lie in $U_{0}$. We write

$$
x_{n}=\left(x_{n}^{(1)}, x_{n}^{(2)}, \ldots, x_{n}^{(i k)}\right) \text {. }
$$

The rectors

$$
x_{0}, x_{1}, \ldots, x_{M K-1}
$$

are the first $M$ terms ${ }^{(2)}$ of the Halton sequenee.

We extend the range of definition of $x_{n}$ over the set of all integers $n$ so as to ensure that

$$
x_{n+H}=x_{n} \text { for every integer } x .
$$

There is, of course, precisely one such extension of the set (3.5).

LFmar 1. Suppose that $I$ is a subinterval of $U_{0}$ of the type

$$
v p_{j}^{-8} \leqslant \beta<(v+1) p_{j}^{-8}
$$

where $v, s$ are integers and $0 \leqslant s \leqslant h$. Then the set of all those $n$ for which $x_{n}^{(j}$ lies in $I$ constitutes a residue class $R$ modulo $p_{j}^{s}$.

Proof. When $s=0$ we must have $I=U_{0}$ and the result is trivial, so we suppose $s>0$. For $0 \leqslant n<\mathbb{H}$ the condition $x^{(j)} \in I$ determines $a_{0}^{(j)}, \ldots, a_{s-1}^{(j)}$ uniquely, but leaves the remaining $a_{p}^{(j)}$ arbitrary. Since $p_{j}^{z}$ is a divisor of the period $M$ appearing in (3.6), the result follows.

In view of the above lemma it is convenient to introduce the following terminology.

DEFINITION. An interval $\left[a_{1}, a_{2}\right)$ is said to be an elementary $p_{j}$ type

$\left({ }^{2}\right)$ Strictly speaking, it is more customary to commence the Halton sequence with $\mathfrak{x}_{1}$ 
interval of order $s$ if $\alpha_{1}, \alpha_{2}$ are consecutive integer multiples of $p_{j}^{-s}$. We reserve the symbol $d$ for elementary intervals.

For every real $t$ we define the set $\Omega(t)=\Omega(h, t)$ in $k+1$. dimensional space by

$$
\Omega(t)=\left\{\left(x_{n}^{(1)}, x_{n}^{(2)}, \ldots, x_{n}^{(l)}, n+t\right) ; n \in \mathbb{Z}\right\} .
$$

In other words, $\Omega(t)$ consists of all points $\left(x_{n}, n+t\right)$ as $n$ ranges through the integers.

Stippose $B$ is a box in $k+1$ dimensional space of the type $\left(I^{(1)} \times I^{(2)} \times \ldots\right.$ $\left.\ldots \times I^{(k)}\right) \times I^{*}$, where $I^{(j)}$ is a subinterval of $U_{0}$ (for $j=1, \ldots, k$ ) and $I^{*}$ is of the type $[0, Y)$ where $Y$ is positive (but otherwise unrestricted). We define $E[\Omega(t) ; B]$ by

$$
E[\Omega(t) ; B]=Z(\Omega(t) ; B)-\nabla(B),
$$

where $Z(\Omega(t) ; B)$ is the number of points of $\Omega(t)$ falling into $B$ and $V(B)$ is the volume of $B$.

4. The basic result. We prove the following result from which Theorem 2 will be deduced in the subsequent section.

Basic Lemara. Let $Y>0$ and, for each $j=1,2, \ldots, k$, let $\eta^{(j)}$ be an integer multiple of $p_{j}^{-h}$ lying in $U_{1}$. Let $B^{*}$ be the box

$$
B^{*}(\eta, Y)=I^{(1)} \times I^{(2)} \times \ldots \times I^{(k)} \times I^{*}
$$

where $I^{(j)}=\left[0, \eta^{(j)}\right)$ and $I_{s}^{*}=[0, \bar{Y})$. Then

$$
\int_{0}^{M}\left|E\left[\Omega(t) ; B^{*}\right]\right|^{2} d t<(4 h)^{k}\left(p_{1} \ldots p_{k}\right)^{2} M .
$$

Proof. If $\eta^{(j)}<1$, it can be seen by considering the expansion of $\eta^{(j)}$ as a decimal in the seale $p_{j}$ that $Y^{(j)}$ can be represented as a union of disjoint elementary $p_{j}$ type intervals of various positive orders not exceeding $h$; the union being such that there are at most $p_{j}-1$ intervals in the union having any given order. If $\eta^{(j)}=1$, we may think of $I^{(j)}$ as being a union consisting of a single elementary interval of order 0 .

Suppose that, in either event, the elementary intervals constituting the union are

$$
J_{1}^{(j)}, J_{2}^{(j)}, \ldots, J_{L_{j}}^{(j)}
$$

of orders $s(j, 1), s(j, 2), \ldots, s\left(j, L_{j}\right)$ respectively, and that the numbering is such that

$$
s(j, 1) \leqslant s(j, 2) \leqslant \ldots \leqslant s\left(j, L_{j}\right) .
$$

In view of our above remarks, the situation is as follows.
LEincs 2. Either $L_{j}=1$ and $s(j, 1)=0$, or

$$
1 \leqslant s(j, 1) \leqslant s(j, 2) \leqslant \ldots \leqslant s\left(j, I_{j}\right) \leqslant h
$$

and there are at most $p_{j}-2$ consecutive equalities in (4.3) (not, of course, counting equalities possibly implicit in $1 \leqslant s(j, 1)$ or $\left.s\left(j, I_{i j}\right) \leqslant h\right)$.

It follows from Lemma 1 that the $n$ for which $x_{n}^{(j)}$ lies in $J_{l}^{(j)}$ form a residue class modulo $x_{j}^{8(j, i)}$; we denote this residue class by $R^{(j)}(l)$.

For any given $l_{1}, \ldots, l_{k}$ satisfying $1 \leqslant l_{j} \leqslant L_{j}\left(j=1, \ldots, l_{i}\right)$, the $n$ for which $x_{n}$ lies in the box

$$
B_{l_{1}, \ldots, l_{k}}=J_{l_{1}}^{(1)} \times J_{l_{2}}^{(2)} \times \ldots \times J_{l_{k}}^{(1)}
$$

constitute the residue class $R(l)$ defined by

$$
R(l)=R\left(l_{1}, \ldots, l_{k}\right)=\bigcap_{j=1}^{k} R^{(j)}\left(l_{j}\right)
$$

Thus, by (2.1), (3.7), (3.8) and the Chinese Remainder Theorem,

$$
\left[E\left[\Omega(t) ;\left(B_{l_{1}, \ldots, l_{k}}\right) \times I^{*}\right]=F^{i}\left[t+R\left(l_{1}, \ldots, l_{k}\right) ; I^{*}\right]\right. \text {. }
$$

It follows from what has been said above that

$$
E\left[\Omega(t) ; B^{*}\right]=\sum_{t_{1}=1}^{L_{1}} \cdots \sum_{l_{k=1}}^{L_{k}} F\left[t+R(\mathbb{l}) ; I^{*}\right] .
$$

Since $I^{*}$ remains fixed throughout the proof of the Basic Lemma, we shall henceforth write simply $F^{\prime}[t+R(t)]$ for $F\left[t+R(t) ; I^{*}\right]$. We now have

$$
\int_{0}^{3 f}\left|E\left[\Omega(t) ; B^{*}\right]\right|^{2} d t=\sum_{i=1}^{L_{1}} \cdots \sum_{l_{k}^{\prime}=1}^{L_{k}} \sum_{l_{1}^{\prime \prime}=1}^{L_{1}} \cdots \sum_{i_{k=1}^{\prime \prime}=1}^{L_{k}} H\left(\boldsymbol{l}^{\prime}, \boldsymbol{t}^{\prime \prime}\right)
$$

where

$$
H\left(l^{\prime}, l^{\prime \prime}\right)=\int_{0}^{M} F\left[t+R\left(l^{\prime}\right)\right] F\left[t+R\left(l^{\prime \prime}\right)\right] d t 。
$$

We consider the integrand on the right hand side of (4.6). For each $j=1,2, \ldots, k$, let

$$
\begin{aligned}
& q_{j}\left(l^{\prime}, b^{\prime \prime}\right)=\min \left(p_{j}^{s\left(j, l_{j}^{\prime}\right)}, p_{j}^{s\left(j, l_{j}^{\prime \prime}\right)}\right), \\
& Q_{j}^{\prime}\left(\dot{c}^{\prime}, z^{\prime \prime}\right)=\max \left(p_{j}^{s\left(j, l_{j}^{\prime}\right)}, p_{j}^{s\left(j, l_{j}^{\prime \prime}\right)}\right),
\end{aligned}
$$

and $\mathcal{A}_{j}\left(l^{\prime}, l^{\prime \prime}\right)=Q_{j}\left(\mathbb{l}^{\prime}, l^{\prime \prime}\right) / q_{j}\left(l^{\prime}, \mathbb{l}^{\prime \prime}\right)$. We note that

$$
A_{j}\left(l^{\prime}, I^{\prime \prime}\right)=p_{j}^{d}, \quad \text { where } \quad a=\left|s\left(j, l_{j}^{\prime}\right)-s\left(j, l_{j}^{\prime \prime}\right)\right| .
$$


Write

$$
M_{j}=M p_{j}^{-h}=\prod_{\substack{i=1 \\ i \neq j}}^{k} p_{i}^{h} \quad(j=1,2, \ldots, k) .
$$

Since the integrand in (4.6) is periodic with period $M$ in $t$, and $M$ is the length of the range of integration, we are entitied (for any integer $a$ ) to replace $t \mathrm{by}$

$$
t+a M_{1} q_{1}\left(t^{\prime}, t^{\prime \prime}\right)
$$

in (4.6). After replacing $t$ by (4.11) in (4.6), we shall sum over the values $(4.12)^{:}$

$$
a=1,2, \ldots, A_{1}\left(\mathbb{l}^{\prime}, \mathbb{Z}^{\prime \prime}\right) \text {. }
$$

We note that, since $\left(M_{1}, p_{1}\right)=1$, as $a$ runs through the set $(4.12)$ the numbers $a M_{1} q_{1}\left(l^{\prime}, l^{\prime \prime}\right)$ represent all the residue classes modulo $Q_{1}\left(l^{\prime}, l^{\prime \prime}\right)$ that are congruent to 0 modulo $q_{1}\left(\boldsymbol{l}^{\prime}, \boldsymbol{l}^{\prime \prime}\right)$.

If $R^{(j)}(l)$ is a residue class modulo $p_{j}^{(j, l)}$ of the type introduced earlier, and $0 \leqslant b \leqslant s(j, l)$, we denote by $R^{(j)}\left(l ; p_{j}^{b}\right)$ the (unique) residue class modulo $p_{j}^{b}$ which contains $R^{(j)}(l)$. We denote by $R_{1}\left(l^{\prime} \mid z^{\prime \prime}\right)$ the modification of the residue class $R\left(l^{\prime}\right)$ obtained by replacing $R^{(I)}\left(l_{1}^{\prime}\right)$ by $R^{(1)}\left(l_{1}^{\prime}\right.$; $\left.q_{1}\left(\boldsymbol{l}^{\prime}, \boldsymbol{l}^{\prime \prime}\right)\right)$ in the representation of $R\left(l_{1}^{\prime}, \ldots, l_{k}^{\prime}\right)$ as an intersection of $h$ residue classes with prime power moduli (whilst leaving the remaining $k-1$ residue classes in the intersection unchanged). Similarly $R_{1}\left(l^{\prime \prime} \mid l^{\prime}\right)$ denotes the modification of the residue class $R\left(l^{\prime \prime}\right)$ obtained by interchanging the roles of $I^{\prime}$ and $l^{\prime \prime}$ above.

Now if $s\left(1, l_{1}^{\prime}\right) \geqslant s\left(1, l_{1}^{\prime \prime}\right)$ the assertion $\left(A^{\prime}\right)$ below is true.

(A') $\sum_{(4.12)} F^{\prime}\left[t+a M_{1} q_{1}\left(l^{\prime}, l^{\prime \prime}\right)+R\left(l^{\prime}\right)\right]=F^{\prime}\left[t+R_{1}\left(l^{\prime} \mid l^{\prime \prime}\right)\right]$ whilst $F\left[t+a M_{1} q_{1}\left(l^{\prime}, l^{\prime \prime}\right)+R\left(d^{\prime \prime}\right)\right]=F^{\prime}\left[t+R_{1}\left(d^{\prime \prime} \mid \delta^{\prime}\right)\right] \quad$ for every $a$.

If $s\left(1, l_{1}^{\prime}\right) \leqslant s\left(1, l_{1}^{\prime \prime}\right)$, the assertion $\left(\mathrm{A}^{\prime \prime}\right)$, obtained from $\left(\mathrm{A}^{\prime}\right)$ by interchanging the roles of $I^{\prime}, l^{\prime \prime}$, is true. (If $s\left(1, l_{1}^{\prime}\right)=s\left(1, l_{1}^{\prime \prime}\right)$ the assertions $\left(A^{\prime}\right)$ and $\left(A^{\prime \prime}\right)$ are of course identical.)

Thus, in any event,

where

$$
A_{1}\left(l^{\prime}, l^{\prime \prime}\right) H\left(l^{\prime}, l^{\prime \prime}\right)=H_{1}\left(l^{\prime}, l^{\prime \prime}\right),
$$

$$
H_{1}\left(\boldsymbol{l}^{\prime}, \boldsymbol{l}^{\prime \prime}\right)=\int_{0}^{M} F\left[t+R_{1}\left(\boldsymbol{l}^{\prime} \mid \boldsymbol{l}^{\prime \prime}\right)\right] F\left[t+R_{1}\left(\boldsymbol{l}^{\prime \prime} \mid \boldsymbol{l}^{\prime}\right)\right] d t .
$$

We now replace $t$ by $t+a M_{2} q_{2}\left(l^{\prime}, l^{\prime \prime}\right)$ in (4.14) and sum over $a=1,2, \ldots$ $\ldots, A_{2}\left(l^{\prime}, l^{\prime \prime}\right)$. We obtain

$$
A_{2}\left(l^{\prime}, l^{\prime \prime}\right) H_{1}\left(l^{\prime}, l^{\prime \prime}\right)=H_{2}\left(l^{\prime}, l^{\prime \prime}\right)
$$

where

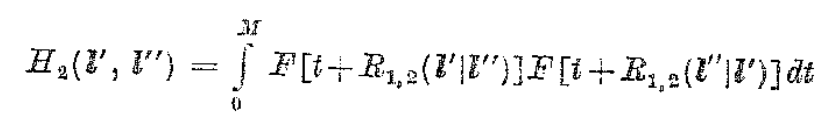

and, for example, $R_{1,2}\left(l^{\prime} \mid l^{\prime \prime}\right)$ is the modification of the residue class $R_{1}\left(l^{\prime} \mid l^{\prime \prime}\right)$ obtained by replacing $R^{(2)}\left(l_{2}^{\prime}\right)$ by $R^{(2)}\left(l_{x}^{\prime} ; q_{2}\left(l^{\prime}, l^{\prime \prime}\right)\right)$ in the representation of $R_{1}\left(l^{\prime} \mid l^{\prime \prime}\right)$ as an intersection of $k$ residue classes with prime power moduli. After $k$ applications of this procedure, we finally obtain

where

$$
H\left(I^{\prime}, I^{\prime \prime}\right)=P^{-1} \int_{0}^{m} F\left[t+R^{\prime}\right] F\left[t+R^{\prime \prime}\right] d t \text {, }
$$

$$
P=\prod_{j=1}^{k} A_{j}\left(l^{r}, l^{\prime \prime}\right)
$$

and $R^{\prime}, R^{\prime \prime}$ are residue classes. We have already noted in $(2.2)$ that $\left|F^{\prime}(t+R)\right|$ $\leqslant 1$ for every real $t$ and every residue class $R$. Thus, by $(4.9),(4.5)$ jields

$$
H I^{-1} \int_{0}^{M}\left|E\left[Q(t) ; B^{*}\right]\right|^{2} d t \leqslant \sigma_{1} \sigma_{2} \ldots \sigma_{k}
$$

where, for $j=1,2, \ldots, k$,

$$
\sigma_{j}=\sum_{i_{j=1}^{\prime}}^{L_{j}} \sum_{l_{j}^{\prime \prime}=1}^{z_{j}} p_{j}^{-\left\{s\left(j, l_{j}^{\prime}\right)-s\left(j, l_{j}^{\prime}\right) !\right.}
$$

We write $\sigma_{j}=\sum_{b} \sigma_{j, b}$, where

$$
\sigma_{j, b}=\sum_{i_{j=1}^{\prime}=1}^{L_{j}} \sum_{\substack{h_{j}^{\prime}=1 \\(4,19)}}^{L_{j}} p_{j}^{-\left\{8\left(j, h_{j}^{\prime}\right)-8\left(j, l_{j}^{\prime \prime}\right)\right\}}
$$

the new condition of summation being

$$
\text { (4.19) } \quad \min \left(s\left(j, l_{j}^{\prime}\right), s\left(j, l_{j}^{\prime \prime}\right)\right)=b \text {. }
$$

If $L_{j}=1$ we have $\sigma_{j}=1$, and if $L_{j}>1$ it follows from Lemma 2 that

$$
\sigma_{j}=\sum_{b=1}^{h} \sigma_{j, b}<2 h\left(p_{j}-1\right)^{2} \sum_{d=0}^{\infty} p_{j}^{-d}<4 h p_{j}^{2} .
$$

Thus (4.17) yields the desired inequality (4.1).

5. Proof of Theorem 2. Let the natural number $N \geqslant 2$ be given, and choose

$$
h=\left[\log _{2} N\right]+1
$$


so that

$$
N \leqslant p_{j}^{h} \quad(j=1,2, \ldots, k) .
$$

For any $\theta$ in $U_{1}^{k}$, and any real $Y$ satisfying

$$
0<Y \leqslant N \text {, }
$$

we use $B^{*}(\theta, Y)$ to denote the Cartesian product of the $k+1$ intervals

Write

$$
\left[0, \theta^{(1)}\right),\left[0, \theta^{(2)}\right), \ldots,\left[0, \theta^{(k)}\right),[0, Y) \text {. }
$$

and

$$
S(t ; \theta, \bar{Y})=E\left[\Omega(t) ; B^{*}(\theta, Y)\right]
$$

$$
T(t ; \theta, Y)=S(t ; \eta(\theta), \bar{Y})
$$

where $\eta=\eta(\theta)$ is defined by taking $\eta^{(j)}$ to be the least integer multiple of $p_{j}^{-h}$ that is not less than $\theta^{(j)}$. Written symbolically,

$$
\eta^{(j)}(\theta)=-p_{j}^{-k}\left[-p_{j}^{h} \theta^{(j)}\right] \quad(j=1, \ldots, k) .
$$

It follows from the Basic Lemma that

$$
\int_{0}^{[M} \int_{U_{1}^{k}} \int_{0}^{N}|T(t ; \theta, Y)|^{2} d t d \theta d Y \ll h^{k} M N,
$$

where the implicit constant depends only on $k$.

For any fixed $t, \theta, Y$, we consider the effect on

$$
S\left(t ; \theta^{(1)}, \theta^{(2)}, \ldots, \theta^{(k)}, Y\right)
$$

of replacing $\theta^{(1)}$ by $\eta^{(1)}(\theta)$, whilst leaving $\theta^{(2)}, \ldots, \theta^{(k)}$ unchanged. On applying (5.4), we must recall the definition (3.8) of $D$. The error introduced in

$$
Z\left(\Omega(t) ; B^{*}\left(\theta^{(1)}, \theta^{(2)}, \ldots, \theta^{(k)}, Y\right)\right)
$$

on replacing $\theta^{(1)}$ by $\eta^{(1)}(\theta)$ does not exceed the number of $n$ in $[-t, \bar{Y}-t)$ for which $x_{n}^{(1)}$ lies in

$$
\left[\eta^{(1)}(\theta)-p_{1}^{-\hbar}, \eta^{(1)}(\theta)\right]
$$

Since this is an interval of the type to which Lemma 1 is applicable it follows from Lemma 1 (with $s=h$ ) in conjunction with (5.2), (5.3) that the difference between

$$
Z\left(\Omega(t) ; B^{*}\left(\eta^{(1)}(\theta), \theta^{(2)}, \ldots, \theta^{(k)}, Y\right)\right)
$$

and $(5.9)$ is at most 1. Again using (5.2), (5.3), we see that the increase in $V\left(B^{*}\right)$ is also at most 1 . Thus the absolute value of the error introduced in (5.8) is also at most 1 .
Clearly, the above argument can also be used to show that the replacement of $\theta^{(2)}$ by $\eta^{(2)}(\theta)$ in (5.10) increases the value of the expression by at most 1 ; and so on. Thus on replacing $\theta^{(1)}, \theta^{(2)}, \ldots, \theta^{(k}$ successively by $\eta^{(1)}(\theta), \eta^{(2)}(\theta), \ldots, \eta^{(k)}(\theta)$ in $(5.8)$, we see that

$$
|S(t, \theta, Y)-T(t, \theta, Y)| \leqslant z
$$

for every $t, \theta, Y$ relevant to $(5.7)$. Since $|S-T| \leqslant k$ implies $|S|^{2} \leqslant 2|T|^{2}+$ $+2 k^{2}$, it follows from (5.7) and (5.1) that there exists a real number ${ }^{*}$, satisfying $0 \leqslant t^{*}<M I$, such that

$$
\int_{U_{1}^{k}} \int_{0}^{N}\left|S\left(t^{*}, \theta, Y\right)\right|^{2} d \theta d Y \ll(\log N)^{k} N .
$$

On recalling (5.4) and the definition (3.7) of $\Omega(t)$, we see from (5.11) that the set

$$
\mathscr{P}=\left\{\left(x_{n}^{(1)}, x_{n}^{(2)}, \ldots, x_{n}^{(k)}, N^{-1}\left(n+t^{*}\right)\right) ; 0 \leqslant n+t^{*}<N\right\}
$$

fulfils the requirements of Theorem 2 .

\section{References}

[1] H. Davenport, Note on irregularities of distribution, Mathematika 3 (1956), pp. 131-135.

[2] J. H. Halton, On the efficiency of certain quasirandom sequences of points in evaluating multidimensional integrals, Num. Miath. 2 (1960), pp. 84-90.

[3] J. H. Halton and S. K. Z aremba, The extreme and $L^{2}$ discrepancies of some plane sets, Monatsch. Math. 73 (1969), pp. 316-328.

[4] J. M. Hammersiey, Honte Carlo methods for solving multivariable problems, Ann. New York Acad. Sei. 86 (1960), pp. 844-874.

[5] K. F. Roth, On irregularities of distribution, Mathematika 1 (1954), pp. 73-79.

[6] - On irregularities of distribution, II, Comm. Pure Appl. Math. 29 (1976), pp. $7 \pm 9-754$.

[7] - On irregularities of distribution, IIT, Asta Arith 35 (1979), pp. 373-384.

[8] 1. V. Vilenkin, Plane nets of integration (in Russian), Z. Vyčisl. Mat. i Mat. Fiz. 7 (1967), pp. 180-196; Engl. transl. in U.S.S.R. Comp. Fiath. and Phys. 7 (1) $(1967)$, pp. 258-267,

IMPERISL COLLEGE

Löndon, S.W. 7, England 